\title{
Predicting the colour associated with odours using an electronic nose
}

\author{
Ryan J. Ward ${ }^{1 *}$
}

Department of Electrical \& Electronic Engineering, University of Liverpool, Liverpool, UK, ryan.ward@liverpool.ac.uk

\section{Shammi Rahman}

Department of Engineering, University of Lincoln, Lincoln, UK

\section{Sophie Wuerger}

Department of Psychology, University of Liverpool, Liverpool, UK

\section{Alan Marshall}

Department of Electrical \& Electronic Engineering, University of Liverpool, Liverpool, UK

Predicting olfactory perception with an electronic nose can aid in the design and evaluation of olfactory-based experiences. We investigate whether the human perception of odours can be predicted outside the bounds of perceived pleasantness and semantic descriptors. We tuned an electronic nose to predict an odour's colour in the CIELAB colour space using human judgements. This revealed that the crossmodal associations people have towards colours could be predicted. Our electronic nose system can predict an odour's colour with a $70-81 \%$ machine-human similarity rating. These findings suggest a systematic and predictable link exists between the chemical features of odours and the colour associated to them. These findings highlight the possibilities of predicting human olfactory perception using an electronic nose.

CCS CONCEPTS $\bullet$ Human-centered computing $\rightarrow$ Interaction design $\rightarrow$ Systems and tools for interaction design; - Applied computing $\rightarrow$ Physical sciences and engineering $\rightarrow$ Engineering $\rightarrow$ Computer-aided design.

Additional Keywords and Phrases: Electronic Nose, Odours, Odour Perception, Regression, Predicting Colours, CIELAB

\section{ACM Reference Format:}

Ryan J. Ward, Shammi Rahman, Sophie Wuerger and Alan Marshall. 2021. Predicting the colour of odours using an electronic nose. In SensoryX '21: Workshop on Multisensory Experiences, together with IMX 2021: ACM International Conference on Interactive Media Experiences. June 21-23, 2021. NY, USA.

Published in accordance with the terms of the Creative Commons Attribution 4.0 International Public License (CC BY 4.0). Permission to reproduce or distribute this work, in part or in whole, verbatim, adapted, or remixed, is granted without fee, provided that the appropriate credits are given to the original work, not implying any endorsement by the authors or by SBC.

2021 Brazilian Computing Society 


\section{INTRODUCTION}

Olfactory perception occurs when our olfactory receptors detect volatile molecules in our nasal cavity. A neural signal is then propagated to our olfactory system forming a semantic, perceptual and physical neural representation of an odour [2]. Humans have thousands of olfactory receptors that are thought to recognise specific chemical features [6]. Like the human olfactory stimulus, electronic noses (e-noses) consist of an array of sensors where the specificity comes from the number of sensors; each sensor has a limited detection capability [11]. E-noses rely on pattern recognition systems to identify and quantify an analyte in the vapour phase [1]. E-noses are predominantly used to detect and discriminate odours [3] and are rarely used for predicting odour perception. This may be because olfactory perception is modulated by both stimulus structure [7] and other perceptual mechanisms including learning, past experience and pleasantness. E-noses are usually fine-tuned to solved specific problems, including but not limited to, lung cancer screening [8] and olfactory visualisation [11] (See [4] for a recent survey on this topic). Crossmodal correspondences can be defined as the consistent associations between stimulus features in different sensory modalities [10]. An example of such associations is between odours: colours and the smoothness of texture [12]. Recently it has been shown that the perceived pleasantness of odours can be predicted using e-nose technology [3,13], highlighting the possibility of predicting crossmodal odour associations using the underlying chemical features. The extent to which these associations can be predicted from the underlying chemical features is still unknown. Robust predictions of crossmodal odour associations would also help in the design process (i.e., perfumers) to create experiences that conform to the user's sensory expectations without extensive psychological tests.

In this paper, we test the hypothesis that human odour perception can be predicted outside the confines of the perceived pleasantness and semantic descriptors. The characteristic response profile generated by an enose can be linked to the colour associated with odours, thereby suggesting that people odour-colour crossmodal correspondences are, at least in part encoded into the molecular properties and therefore capturable by a machine.

\subsection{L*a*b* Colour Space}

The $L^{*} a^{*} b^{*}$ colour space, also referred to as the CIELAB colour space, expresses colour as three channels: $L^{*}$ for perceptual lightness between the values of $0-100$, $a^{*}$ red - green with values typically ranging from -127 to 127 , and $b^{*}$ yellow - blue again with values typically ranging from -127 to 127 . The $L^{*} a^{*} b^{*}$ colour space was designed to be perceptually uniform where a numerical change in colour aligns with a similar perceptual change (see [9] for more information).

\section{MATERIALS \& METHODS}

\subsection{Perceptual Data}

In our prior work [12], we explored a range of olfactory crossmodal correspondences. One of these was the perceived colour of odours. Sixty-eight participants were presented with ten unlabelled odours (black pepper, caramel, cherry, coffee, freshly cut grass, lavender, lemon, orange, peppermint and pine). Participants were placed in a lightproof anechoic chamber equipped with an overhead luminaire (GLE-M5/32; GTI Graphic Technology Inc., Newburgh, NY). The lighting in the room was kept consistent by using the daylight simulator of the overhead luminaire. Participants were asked to select a colour most closely corresponded to the current 
odour by freely selecting a colour from the $L^{*} a^{*} b^{*}$ colour space. Participants could slide through this space by adjusting the $L^{*}$ channel via the use of a slider. The $L^{*}, a^{*}$ and $b^{*}$ of their final selection were saved.

\subsection{Electronic Nose}

To extract the chemical features from odours, we used a custom-made e-nose, as reported in [11]. We added an MQ9 gas sensor and changed the container to a custom 3D printed and sealable container made using PLA. The gas sensors used in the e-nose are as follows: MP503, BME680, MQ3, MQ5, MQ9 and a WSP2110. The e-nose was controlled using custom software, where the raw sensor data is sent to a nearby computer via a UDP connection. Any out of order packets were put back into order before any pre-processing. The e-nose recordings have 11 features - time, air quality, pollution level, temperature, pressure, humidity, gas, MQ3, MQ5, MQ9 and HCHO. An image of the e-nose experimental setup is shown in Fig. 1.

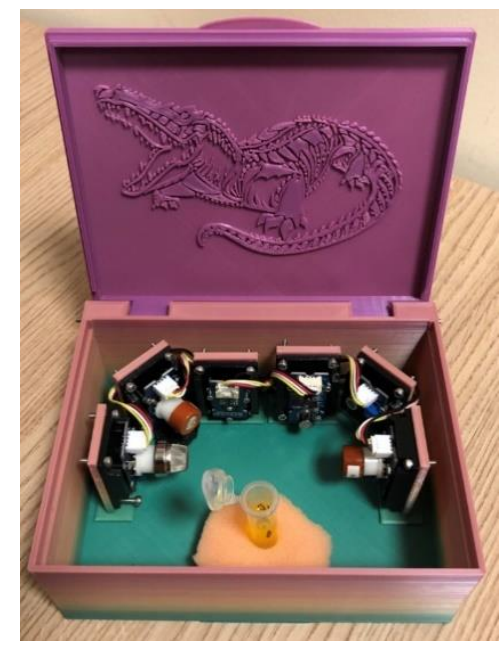

Figure 1: Photo of the experimental setup for the recording of different odours.

\subsection{Chemical Data}

Odours were prepared in the same manner as reported in [12], using the same volume $(4 \mathrm{~mL})$ and brand when the perceptual data was initially collected. Odours were placed inside the e-nose, the lid was closed and recorded for 10 minutes, the container was flushed with ambient air for a minimum of 30 minutes between recordings. We presented the odours to e-nose in the same fashion as they were presented to the participants. We did this because factors such as temperature and intensity can bias people's crossmodal associations. A total of 100 recordings were prepared for our experiments, with ten recordings for each odour. Before odours were used in the analysis the sensor responses were first pre-processed by taking the mean over 1-second intervals turning an $\mathrm{N} \times 10$ matrix into a $600 \times 10$ matrix. Each sensor signal was then smoothed using a moving average filter; afterwards, the median value for each of the sensor's response was used for the regression, resulting in a $100 \times 10$ matrix.

\subsection{Data Analysis}

We created the graphs and performed the data analysis using MATLAB ${ }^{\mathrm{TM}} \mathrm{R} 2018 \mathrm{~b}$. The recordings obtained for the data analysis consisted of 11 features. The first feature was the time component of when the packets 
were sent allowing for our software to reorder the packets and take the mean over one second intervals. The time component was removed after pre-processing and was not used in the training or testing of the regression models. All odour samples were placed in the same location when recording to negate distance-based sensor bias.

\section{RESULTS \& DISCUSSION}

We first measured ten odours using the e-nose outlined in Section 2.2. Each odour was measured ten times at the same volume $(4 \mathrm{~mL})$ and using the same brand of essential oil; to align the odour recordings with the perceptual data. Example sensor responses are shown in Fig. 2.
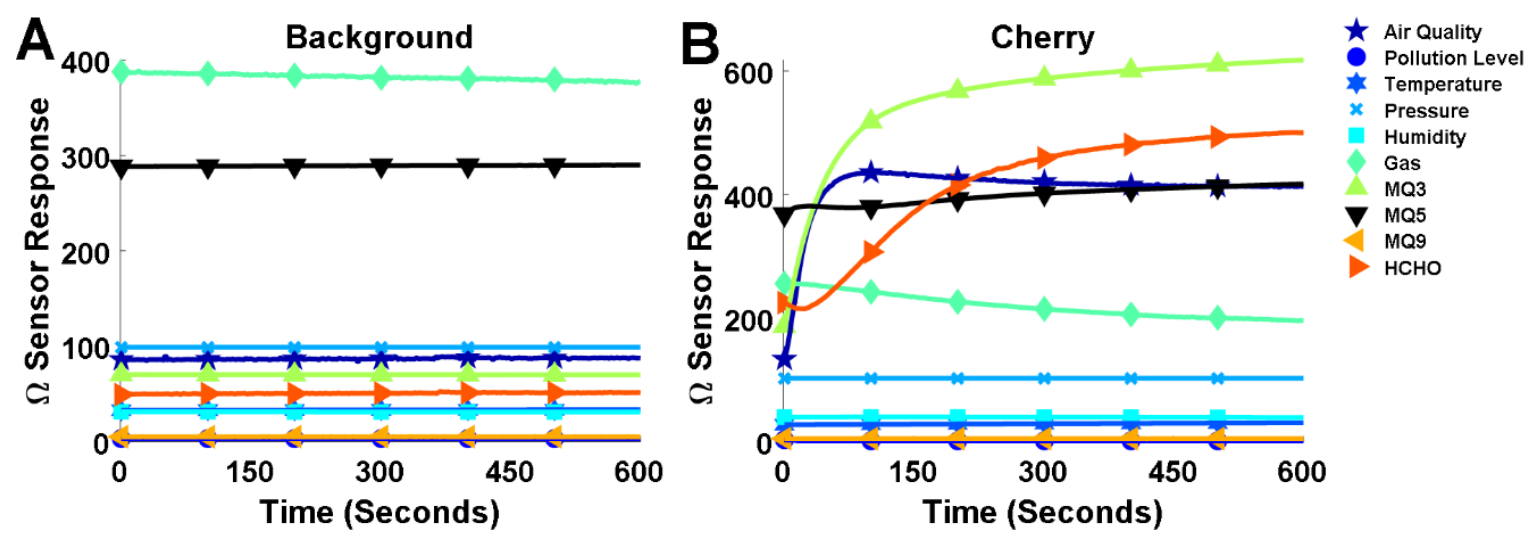

Figure 2: Example sensor responses showing (A) sensor response over time for no odour and (B) sensor responses over time for cherry essential oil. Some sensor responses will be minimal (i.e., temperature and humidity).

We used ten of the features provided by the e-nose for the training and testing for the regression models; the time component feature was removed after pre-processing. To prevent sensor bias, we standardised the input by centreing using the feature mean and scaled using the standard deviation. We then constructed a response vector by converting the participant selected colours back into $L^{*} a^{*} b^{*}$ space. The outliers were removed for each channel independently by replacing any value outside the range of \pm 1.5 std with the channel's mean, excluding the detected outliers. Each response vector was then repeated ten times so that each recording would have a response value. We then trained, tested, and compared different regression models to determine which algorithm would give us the lowest root squared mean error (RSME). The results are shown in Table 1.

Table 1: Results for the comparison of the regression algorithms.

\begin{tabular}{cccc}
\hline Algorithm & $\begin{array}{c}\mathrm{L}^{*} \\
\text { (RSME) }\end{array}$ & $\begin{array}{c}\mathrm{a}^{*} \\
(\mathrm{RSME})\end{array}$ & $\begin{array}{c}\mathrm{b}^{*} \\
(\mathrm{RSME})\end{array}$ \\
\hline Linear Regression & 4.83 & 7.15 & 11.01 \\
Support Vector Machine & 1.31 & 4.08 & 5.61 \\
Random Forest & 3.12 & 6.49 & 3.88 \\
Gaussian Process & 1.06 & 3.5 & 3.4 \\
Regression & & & \\
\hline
\end{tabular}


From Table 1 we can see that Gaussian Process Regression (GPR) gives the lowest RMSE for all three predicted attributes $\left(L^{*}, a^{*}, b^{*}\right)$. Therefore, we decided to optimise GPR parameters for each of our three models $\left(L^{*}, a^{*}\right.$ and $\left.b^{*}\right)$. To determine the robustness of the developed models, we used an approach leaving out one odour. We trained each model 10 times, using all the recordings for nine odours and left all the recordings for one odour aside for testing. This resulted in a $90 \times 10$ matrix for training and a $10 \times 10$ matrix for testing. We then used a Pearson correlation to compare the GPR models to the perceptual data (predicted vs actual). We first computed the human-human correlation between the 68 participants who reported the colour correspondences and each colour channel's median values. This revealed a correlation of $(r=0.5676, p<$ $0.0001)$ for the $L^{*}$ channel, $(r=0.5662, p<0.0001)$ for the $a^{*}$ channel and $(r=0.5990, p<0.0001)$ for the $b^{*}$ channel. Next, we took the correlation between the median ratings and the machine regression ratings (predicted vs. actual) revealing a correlation of $(r=0.49, p<0.0001)$ for the $L^{*}$ model, $(r=0.44, p<0.0001)$ for the $a^{*}$ model and $(r=0.42, p<0.0001)$ for the $b^{*}$ model. As shown in Fig. 3.
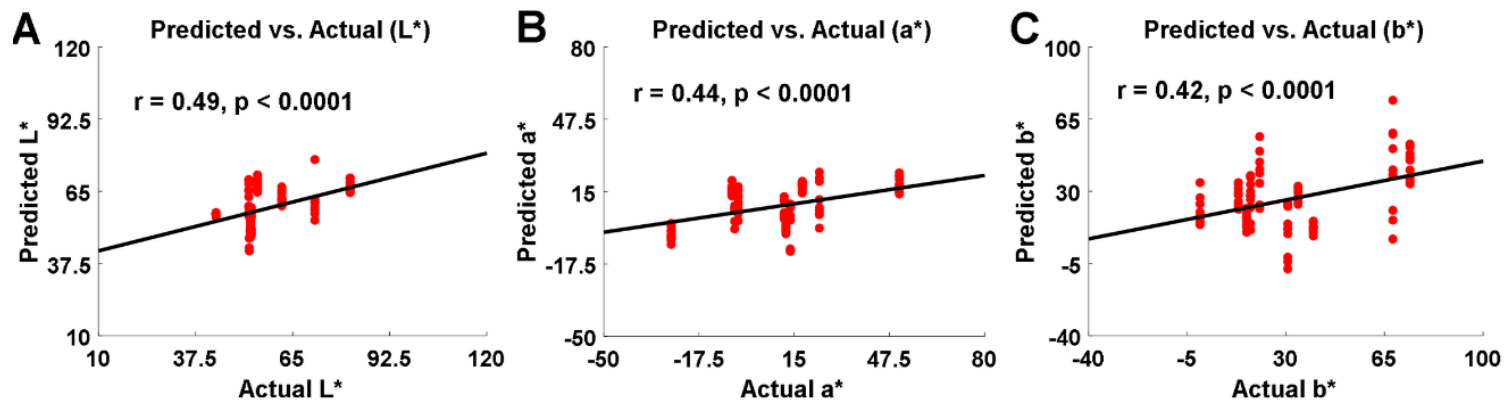

Figure 3: Predicted vs. actual plots for: (A) the $L^{*}$ colour channel, (B) the $a^{*}$ colour channel and (C) the $b^{*}$ colour channel.

We then calculated the percentage of the human-machine correlation relative to the human-human correlation for each of the colour channels: for the $L^{*}$ channel a correlation of $86.33 \%$ was obtained $(0.49 /$ $0.5676 * 100=86.33 \%)$, for the $a^{*}$ channel a correlation of $78.66 \%$ was obtained $(0.44 / 0.5622 * 100=78.66 \%)$, and, for the $b^{*}$ channel a correlation of $70.11 \%$ was obtained $(0.42 / 0.5990 * 100=70.11 \%)$. These values were calculated in this manner to align with the relevant prior literature [3,13]. Overall, these results support our hypothesis that there is a predictable link between the colours associated to odours and their chemical features. Predominantly these results show that e-noses can capture, model, and predict the crossmodal perception of odours. However, the extent of this remains to be investigated.

\section{CONCLUSION}

In this paper, we have used the underlying chemical features of odours to design and create a model to predict people's colour correspondences towards odours. We split the colours in the $L^{*} a^{*} b^{*}$ colour space, producing three separate channels ( $\mathrm{L}^{*}$ - lightness), $\mathrm{a}^{*}$ (red - green) and $\mathrm{b}^{*}$ (yellow - blue). The machine-human correlations were $86.33 \%$ for the $L^{*}$ channel, $78.66 \%$ for the $a^{*}$ and $70.11 \%$ for the $b^{*}$ channel of the human-human correlations. Thus, highlighting the possibility of using e-noses to predict human olfactory perception and implying that the colour associated with odours are partly written into the molecular properties of the stimulus [5]. We believe the models created in this paper could potentially help in the design of olfactory based products and experiences. An important limitation to our claim is that only a portion of olfactory perception is hard-wired 
and innate, which our findings reflect. Olfactory perception can also be modulated or influenced by various mechanisms, including culture, context, expectations, experience, and multisensory convergence [7].

Future work could include determining the extent to which the crossmodal perception of odours can be predicted (e.g., perceived smoothness, musical genres, and the perceived angularity of odours). The findings reported here could be improved upon by including a larger sample size of odours and the colours people associate with them. The potential of predicting human perception is not limited to odours; it may also be possible to predict crossmodal correspondences attributed to taste; this could be done by using an e-tongue.

\section{ACKNOWLEDGMENTS}

This work was funded by the Engineering and Physical Sciences Research Council (EPSRC) under grant number EP/P004016/1.

\section{REFERENCES}

[1] Michael C. Burl, Brett J. Doleman, Amanda Schaffer, and Nathan S. Lewis. 2001. Assessing the ability to predict human percepts of odor quality from the detector responses of a conducting polymer composite-based electronic nose. Sensors Actuators, $B$ Chem. 72, 2 (2001), 149-159. DOI:https://doi.org/10.1016/S0925-4005(00)00645-6

[2] A. Fournel, C. Ferdenzi, C. Sezille, C. Rouby, and M. Bensafi. 2016. Multidimensional representation of odors in the human olfactory cortex. Hum. Brain Mapp. 37, 6 (2016), 2161-2172. DOI:https://doi.org/10.1002/hbm.23164

[3] Rafi Haddad, Abebe Medhanie, Yehudah Roth, David Harel, and Noam Sobel. 2010. Predicting odor pleasantness with an electronic nose. PLoS Comput. Biol. 6, 4 (2010). DOI:https://doi.org/10.1371/journal.pcbi.1000740

[4] Diclehan Karakaya, Oguzhan Ulucan, and Mehmet Turkan. 2020. Electronic Nose and Its Applications: A Survey. Int. J. Autom. Comput. 17, 2 (2020), 179-209. DOI:https://doi.org/10.1007/s11633-019-1212-9

[5] Sarah E. Kemp and Avery N. Gilbert. 1997. Odor intensity and color lightness are correlated sensory dimensions. Am. J. Psychol. 110, 1 (1997), 35-46. DOI:https://doi.org/10.2307/1423699

[6] F. Kermen, A. Chakirian, C. Sezille, P. Joussain, G. Le Goff, A. Ziessel, M. Chastrette, N. Mandairon, A. Didier, C. Rouby, and M. Bensafi. 2011. Molecular complexity determines the number of olfactory notes and the pleasantness of smells. Sci. Rep. 1, (2011), 1-6. DOI:https://doi.org/10.1038/srep00206

[7] Rehan M. Khan, Chung Hay Luk, Adeen Flinker, Amit Aggarwal, Hadas Lapid, Rafi Haddad, and Noam Sobel. 2007. Predicting odor pleasantness from odorant structure: Pleasantness as a reflection of the physical world. J. Neurosci. 27, 37 (2007), 1001510023. DOI:https://doi.org/10.1523/JNEUROSCI.1158-07.2007

[8] Wang Li, Hongying Liu, Dandan Xie, Zichun He, and Xititan Pi. 2017. Lung Cancer Screening Based on Type-different Sensor Arrays. Sci. Rep. 7, 1 (2017). DOI:https://doi.org/10.1038/s41598-017-02154-9

[9] Ronnier Luo. 2016. Encyclopedia of color science and technology. Springer Publishing Company, Incorporated.

[10] Charles Spence. 2011. Crossmodal correspondences: A tutorial review. Attention, Perception, Psychophys. 73,4 (2011), $971-995$. DOl:https://doi.org/10.3758/s13414-010-0073-7

[11] Ryan J. Ward, Fred P.M. Jjunju, Elias J. Griffith, Sophie M. Wuerger, and Alan Marshall. 2020. Artificial Odour-Vision Syneasthesia via Olfactory Sensory Argumentation. IEEE Sens. J. 21, 5 (2020), 6784-6792. DOI:https://doi.org/10.1109/JSEN.2020.3040114

[12] Ryan J. Ward, Sophie Wuerger, and Alan Marshall. 2020. Smelling sensations: olfactory crossmodal correspondences. bioRxiv (January 2020), 2020.04.15.042630. DOI:https://doi.org/10.1101/2020.04.15.042630

[13] Danli Wu, Yu Cheng, Dehan Luo, Kin Yeung Wong, Kevin Hung, and Zhijing Yang. 2019. POP-CNN: Predicting odor's pleasantness with convolutional neural network. IEEE Sensors 19, 23 (2019), 11337-11345. 